exacerbate many symptoms of ADHD, impair scholastic performance and cause memory problems.

Aims We aimed to analyse the prevalence of ADHD and sleep problems among children and young people seen within local Community Child Health clinics of a Scottish NHS Trust over a 12 month period.

Methods A retrospective review of all patients seen in the outpatient clinics between June 2016 and May 2017 in two different clinics within NHS Fife was carried out. ADHD was diagnosed using validated Swanson, Nolan, and Pelham -IV Questionnaire (SNAP-IV). Sleep problems were diagnosed empirically from detailed clinical history provided by the parents/carers and patients.

Results 93 (17\%) out of 543 children reviewed had ADHD. Prevalence of sleep difficulties was higher among the ADHD children (52\%) compared to $30 \%$ in the whole cohort. A significantly higher proportion of ADHD patients with sleep problems were on treatment with Melatonin compared to those without ADHD (75\% vs 56\%).

There was a statistically significant association between the degree of socioeconomic deprivation and the prevalence of ADHD and or sleep difficulties. The proportion of children with either or both ADHD and sleep difficulties living in the most deprived (Quintile 1) areas was four times higher than those from the most affluent areas (11.2\% vs $2.8 \%)$.

Conclusion Identification and effective management of daytime sleepiness, sleep-disordered breathing or problematic behaviours at bedtime and upon awakening at night can profoundly improve the symptoms of ADHD in children and adolescents. Evidence-based guidelines for the management of sleep problems in children with ADHD are urgently needed.

\section{G468 ADHD MANAGEMENT - A QUALITY IMPROVEMENT PROJECT}

B Khan, G Rajendran, N Sylvester, K Ganjam, P Mani. Community Paediatrics, Mid Yorkshire Hospitals NHS Trust, Wakefield, UK

10.1136/archdischild-2018-rcpch.456

Attention Deficit Hyperactivity Disorder has an incidence of $3 \%-5 \%$ among school-going children; $60 \%$ of which will continue to have symptoms into adulthood. The aim of this project was to assess all aspects of service delivery to these children as per NICE guidelines at Mid Yorkshire Hospitals NHS Trust.

Methods The first cycle was run retrospectively in September -November 2016. An audit tool was used that reviewed children attending clinics in August 2016. Patients with a diagnosis after 1 st January 2011 were included. The patients were reviewed for details at time of diagnosis or management.

Following the audit, a form was developed to ensure that essential information was documented properly. The unit liaised with local services to improve parent and children workshops availability. A re-audit was run for patients attending in August 2017 using the same parameters to complete the audit loop.

Results 74 patients were included in 2016, while 50 patients have been studied in 2017 (table 1).

\begin{tabular}{lll} 
Abstract G468 Table 1 & & \\
\hline Details at diagnosis & $\mathbf{2 0 1 6}$ & $\mathbf{2 0 1 7}$ \\
\hline Developmental history & $90 \%$ & $100 \%$ \\
Mental state assessment & $74 \%$ & $100 \%$ \\
Observer report used & $100 \%$ & $100 \%$ \\
Abnormal score in observer report & $96 \%$ & $100 \%$ \\
Special education needs noted & $27 \%$ & $59 \%$ \\
Parental training offered & $50 \%$ & $78 \%$ \\
Child social skills training offered & $31 \%$ & $82 \%$ \\
Drug treatment & $100 \%$ & $63 \%$ \\
Baseline weight and height plotted & $100 \%$ & $100 \%$ \\
Baseline blood pressure noted & $63 \%$ & $92 \%$ \\
Baseline heart rate noted & $17 \%$ & $33 \%$ \\
Cardiovascular examination documented & $53 \%$ & $52 \%$ \\
Blood pressure noted at review & $10 \%$ & $72 \%$ \\
Treatment of co-existing conditions at & $100 \%$ & $100 \%$ \\
review & & \\
Side effects at review & $100 \%$ & $100 \%$ \\
\hline
\end{tabular}

Conclusion The project reveals improvement in compliance with NICE guidelines, particularly for monitoring of patients $\mathrm{BP}$ on drug treatment, as well as better education for parents and children. However, parental training and child social skills training are not available to all families due to limited resources.

\section{G469 IMPACT OF CAPSS AND BPSU IN IDENTIFYING THE IMMINENT NEEDS OF ADHD PATIENTS IN TRANSITION FROM CHILDRENS TO ADULTS SERVICES ACROSS THE UNITED KINGDOM}

1,2,3 H Ayyash, ${ }^{2,4} \mathrm{TJ}$ Ford, ${ }^{4} \mathrm{~A}$ Janssens, ${ }^{4} \mathrm{H}$ Eke, ${ }^{4} \mathrm{~A}$ Price, ${ }^{3} \mathrm{M}$ Morton, ${ }^{2,3} \mathrm{R}$ Lynn. ${ }^{1}$ Integrated Neurodevelopmental Children's Services, Cambridgeshire and Peterborough NHS Trust, Peterborough, UK; ${ }^{2}$ Child and Adolescent Surveillance System (CAPSS), RCPsychiatry, London, UK; ${ }^{3}$ British Paediatric Surveillance Unit, RCPCH, London, UK; ${ }^{4}$ Child Mental Health Group, Institute of Health Research, University of Exeter Medical School, Exeter, UK

\subsection{6/archdischild-2018-rcpch.457}

Background The Child and adolescent Psychiatry Surveillance System(CAPSS) is the British Paediatric Surveillance Unit's (BPSU's) baby sibling. It was established in year 2009, and to date has completed more than 6 National studies on children's mental health, with one more in the field of trnsition in ADHD.

Aim 1 st: To dissminate the clinically important findings from the National Transitional ADHD study run on the CAPSS and the BPSU in the UK.

2nd: To raise awareness of CAPSS methodology and its relevance for clinical practice for clinicians and for researchers working with people across the life span.

Methods This ADHD surveillance study run in collaboration with the Child and Adolescent Psychiatric Surveillance System (CAPSS) and British Paediatric Surveillance Unit(BPSU).Each month these units mailed a tick box response card to all consultant paediatricians and child psychiatrists in the UK. Consultants reported on the number of young patients who needed ongoing medications for ADHD and is within six 
months of the age boundary for their service. Baseline questionnaire was utilised to confirm the eligibility of the case, current treatment and comorbidity. Additional data were collected on the adult service to whom they were referred. Consultants were also asked to evaluate the different aspects of an optimal transition.

The BPSU reported 135 cases with 64 returned questionnaires and 51 eligible ADHD cases in transition. The CAPSS reported on 115 cases with 47 returned questinnaires and 39 eligible ADHD cases in need of transition were identified.

Results from the surveillance period which ended in November 2016 are very exciting. In general they indicate poor transition processes for young people with less than $25 \%$ of clinicians holding a transition planning meeting or having a handover period and less than 50\% having the referral to an adult mental health service accepted.

Conclusions Both paediatricians and child psychiatrists are equally involved in the management of young children with ADHD. However, there are still gaps in the provision of transitions of ADHD adolescents across the lifespan in most UK regions.

National guidelines that aims to promote optimal transitions and reduce perceived barriers to transition of care for young people with ADHD out of children's to adult services should be established.

Funding acknowledgement This project was funded by the National Institute for Health Research - Health Services and Delivery Research programme (project ref: 14/21/52)

\section{G470 CO-OCCURRENCE AND CO-MORBIDITIES AMONG CHILDREN AND ADOLESCENTS WITH ADHD AND ASD IN A SCOTTISH LOCAL AUTHORITY}

MO Ogundele. Community Paediatrics Unit, NHS Fife, Glenwood Health Centre, Glenrothes, $U K$

\subsection{6/archdischild-2018-rcpch.458}

ADHD is the commonest childhood neuro-behavioural disorder, affecting $5 \%$ to $12 \%$ of school-age children and about $1 \%$ of children meet the criteria for ASD. Co-occurrence of ASD and ADHD is increasingly recognised after introduction of DSM-5. They both share difficulties with emotional control, attention and high levels of negative affect, with differing underlying motivational and behavioural tendencies.

Objectives We analysed the clinical characteristics of children and young people with ADHD and ASD audited within two Community Child Health clinics of a Scottish NHS Trust over a 12 month period.

Methods A retrospective review of all patients seen in the outpatient clinics between June 2016 and May 2017 within an NHS Region was carried out. ADHD was diagnosed using validated Swanson, Nolan, and Pelham -IV Questionnaire (SNAP-IV). Sleep problems were diagnosed empirically from detailed clinical history provided by the parents/carers/patients. ASD was diagnosed by a multidisciplinary approach involving detailed assessment individually by the Educational Psychologist, Clinical Psychologist, Speech and Language Therapist and the Community Paediatrician, followed by group discussion, using the ICD-10 checklist criteria to confirm or refute a Diagnosis.

Results ASD and ADHD constituted 13\% and 17\% of the clinic caseloads respectively. They presented with similar characteristics including male gender preponderance $(4.3: 1$ and $4.5: 1)$, proportion of new referrals $(20 \%$ and $18 \%)$ and discharges (22\% and 19\%) respectively. 19\% of ASD children had ADHD while 14\% of ADHD children had ASD. They had a similar range of co-morbidities but sleep, emotional problems and DCD were commoner among ADHD patients (52\%, 25\% and $19 \%$ vs $44 \%, 17 \%$ and $9 \%$ respectively). CAMHS and General Paediatricians were more commonly involved with ADHD patients $(33 \%$ and $14 \%$ vs $20 \%$ and 6\%) than ASD children.

ASD patients were equally distributed between young (59 years) and older school age (10-14 years) children (20\% and $18 \%$ respectively) while most ADHD patients were chiefly older (10-14 years). ADHD patients were on average 20 months older, attended more clinics (average 2.2 vs 1.6 ) and had higher number of co-morbidities (average 2.3 vs 1.6 ).

Table 1 summarises the clinical and epidemiological characteristics of children and adolescents with ADHD and ASD.

\section{Child Health Ethics and Law Special Interest Group}

\section{G471 MORAL DISTRESS, TRAUMA AND BURNOUT IN STAFF IN RELATION TO CHANGES IN PICU OUTCOMES, CHALLENGING CASES AND MEDIA INVOLVEMENT IN DISAGREEMENTS ABOUT END-OF-LIFE CARE}

${ }^{1} \mathrm{G}$ Colville, ${ }^{2} \mathrm{M}$ Rutt, ${ }^{3} \mathrm{Z}$ Berger, ${ }^{3} \mathrm{P}$ Titman, ${ }^{2,4} \mathrm{~J}$ Brierley. ${ }^{1}$ Paediatric Psychology Service, St George's University Hospitals NHS Foundation Trust, London, UK; ${ }^{2}$ Neonatal Intensive Care Unit, Great Ormond Street Hospital, London, UK; ${ }^{3}$ Paediatric Psychology Department, Great Ormond Street Hospital, London, UK; ${ }^{4}$ Department of Paediatric Bioethics, Great Ormond Street Hospital, London, UK

\subsection{6/archdischild-2018-rcpch.459}

Background Technological advances have decreased PICU mortality but increased the number of children surviving with disability or technologically-dependent. Death in PICU most frequently follows withdrawal of life-sustaining therapy (LST), increasingly after prolonged admissions for invasive organ support. Disagreements with families about cessation of life-sustaining therapy (LST) can be protracted, distressing for everyone, harmful to the child and ultimately require court adjudication. Little is known about the impact of this, or of that of social/other media campaigns when families decides to involve the press, as is increasingly the case

Method 50 staff (39 nurses; 9 doctors and 2 AHPs) were surveyed using the Moral Distress Scale-Revised (MD-R) questionnaire ${ }^{1}$; Trauma Screening Questionnaire (TSQ) and abbreviated Maslach Burnout Inventory (aMBI) together with several open-ended questions about their experiences and what they felt had been learned 3 months after the final court decision following one such high profile case.

Results Mean moral distress score (MDS-R) was 96, consistent with recent Canadian multi-centre PICU study and higher than average Adult ICU scores (57-83 in the literature). Specifically, in relation to the recent high profile case $15 \%$ scored in the clinically significant range for post- traumatic stress symptoms (TSQ); a significant number reported a number of sub-clinical symptoms; $68 \%$ reported being 'upset by reminders of the event' and 53\% reported 'heightened awareness of potential danger' to themselves and others at least 
twice in the previous week. Burnout symptoms (aMBI): 44\% reported emotional exhaustion at a high level; 17\% reported a high level of depersonalization.

The $25 \%$ of staff considering leaving PICU reported higher burnout (emotional exhaustion) scores $(p=0.001)$; higher posttraumatic stress scores $(p=0.04)$ and were also less likely to feel supported in relation to moral distress $(p=0.010)$.

Conclusions These findings highlight the impact of changing PICU outcomes on staff, with increased survival of children with severe disability or technologically-dependent; and death following prolonged PICU admission sometimes after disagreements about cessation of LST, occasionally with public campaigns. They illustrate that the dimension of moral distress is one which needs to be acknowledged, along with burnout and post-traumatic stress ${ }^{2}$ in a comprehensive assessment of staff well-being.

\section{REFERENCES}

1. Hamric $A B$, Borchers $C T$, Epstein EG. Development and testing of an instrument to measure moral distress in healthcare professionals. AJOB Prim Res 2012;3:1-

2. Colville GA, Smith JG, Brierley J, et al.Coping with staff burnout and work-related posttraumatic stress in intensive care. Pediatr Crit Care Med 2017;18:e267-e273.

\section{G472 YOUNG PEOPLE'S VIEWS ON ACCESSING AND USING PERSONAL RECORDS FOR RESEARCH PURPOSES}

${ }^{1} \mathrm{R}$ Mistry, ${ }^{1,2} \mathrm{M}$ Emonts, ${ }^{1,2} \mathrm{R}$ Agbeko, ${ }^{3}$ Young Person's Advisory Group, ${ }^{4} \mathrm{~L}$ Wilson, ${ }^{1} \mathrm{M}$ Walsh, 'EJ Lim. 'Paediatrics, Great North Children's Hospital, Newcastle upon Tyne, UK; ${ }^{2}$ Institute of Cellular Medicine, University of Newcastle, Newcastle upon Tyne, UK; ${ }^{3}$ Young Person's Advisory Group North East, Newcastle upon Tyne, UK; ${ }^{4}$ Connected Health Cities, Newcastle upon Tyne, UK

\subsection{6/archdischild-2018-rcpch.460}

Aim Modernisation of records, coupled with advances in informatics, is making analyses of electronic records an increasingly rich source for researchers to help form evidence-based policy and treatments.

We aimed to review the public's views on health research from electronic records and databases, with a focus on children and young people (CYP).

Method

- A literature search on the public's perceptions of research from health records, data linkage, and acceptability of different modes of consent.

- Two focus groups using semi-structured discussions, to explore young people's perceptions of access to healthcare data, were held with our Young Person's Advisory Group. YPAG consists of 30 12-17 year-olds from diverse socioeconomic and ethnic backgrounds.

Results Published research is primarily in adults. There is widespread unawareness and misunderstanding of research using health records. The public wish to be informed when their data is used for research as a matter of courtesy and to allow control of their participation. Opt-in consent was preferred. Opt-out consent is deemed potentially acceptable, with some studies demonstrating increased acceptability when participation bias is explained. Only one study explored CYP's views (17-19 year-olds). This broadly mirrored adults' views, including attitudes towards opt-out consent. No studies were found involving younger adolescents.

Our focus groups highlighted young peoples' strong sense of ownership of their data and desire to be informed.
Acceptability of access to data varies depending on exactly what is being used, by whom and for what purpose. A high degree of precision and flexibility in permission-granting was desired, alluding to dynamic consent. A distrust in the security of their data co-existed with a misunderstanding of the protection anonymization provides.

Conclusion CYP are clear in their desire for active engagement when it comes to using their data for research and show appreciation for balancing one's privacy against the 'greater good' for society. Further work is needed to explore how best to inform CYP of data use, allow informed participation, tackle misconceptions and maximise their partnership in this type of research. This is required to increase researchers' transparency and accountability with the public and maintain public trust in clinical research.

\section{G473 A REVIEW OF THE NEUROLOGY CONSULTS OF THE CLINICAL ETHICS SERVICE AT A TERTIARY CHILDREN'S HOSPITAL}

${ }^{1}$ A Horne, ${ }^{2} \mathrm{P}$ Prabhakar, ${ }^{3} \mathrm{~J}$ Brierley, ${ }^{2,3}$ S Aylett. ' University College London, London, UK; ${ }^{2}$ Department of Paediatric Neurology, Great Ormond Street Hospital, London, UK; ${ }^{3}$ Department of Paediatric Bioethics, Great Ormond Street Hospital, London, UK

\subsection{6/archdischild-2018-rcpch.46}

Background The medical care of children with severe neurological disease, poses a number of ethical challenges; perhaps due to the lack of a cure, use of experimental treatment, and life limiting nature. When such ethical dilemmas arise, the clinical team and family may benefit from Clinical Ethics Support to help a consensus emerge on the best course of action for the child.

At this centre, a Rapid Case Review Service (RCRS) can occur within hours, but frequently days to review issues with the child's multidisciplinary team and parents. Little research has been carried out on this cohort's ethical challenges, and use ethics.

Objectives Understand medical, ethical and legal aspects of neurology RCRS consult and its role in addressing ethical problems faced

Methods Of 44 RCRS between 2012-2016, 11 were identified as having had a primary neurological disorder

Data obtained from RCRS referral form; Meeting minutes and Electronic patient records then categorised into 4 main variables: Demographics, Medical, Ethical and Legal. Confidentiality upheld by removing any patient identifiers

Results Demographics: Age 1-16 years (median 9);

Medical: Average of 4 clinical diagnoses (range 2-5) Main categories: Neurolgical (5) (cerebral palsy and hydrocephalus); Neuro-metabolic (Leigh's syndrome, Pompe disease); neuromuscular (DMD, SMA 1). Support teams: Palliative Care (10); External (8); Social Care (8); Psychology (6). Other concurrent conditions: Epilepsy (4); Neurodevelopmental delay (2); Learning disability (3). All had life limiting conditions. 4 had died by time of review ( 3 treatment withdrawal); 6 returned home of which 2 were now attending school.

Ethical issues:

- Conflicts between the clinical team and parents (6)

- RCRS recommendations: Involve palliative care and discuss DNAR; Seek second opinions regarding management; create a care plan with parents' involvement 